\title{
A multi-centre, randomised, double-blind, placebo-controlled clinical trial of methylphenidate in the initial treatment of acute mania (MEMAP study)
}

Ulrich Hegerl ${ }^{a}$,*, Roland Mergl ${ }^{a}$, Christian Sander ${ }^{a}$, Jens Dietzel ${ }^{a}$, Istvan Bitter ${ }^{\mathrm{b}}$, Koen Demyttenaere ${ }^{\mathrm{c}}$, Ricardo Gusmão ${ }^{\mathrm{d}, \mathrm{e}}$, Ana González-Pinto ${ }^{\mathrm{f}, \mathrm{g}}$, Iñaki Zorrilla ${ }^{\mathrm{f}, \mathrm{g}}$, Adriana García Alocén ${ }^{\mathrm{f}, \mathrm{g}}$, Victor Perez Sola ${ }^{\mathrm{g}, \mathrm{h}}$, Eduard Vieta ${ }^{\mathrm{g}, \mathrm{i}}$, Georg Juckel ${ }^{\mathrm{j}}$, Ulrich S. Zimmermann ${ }^{\mathrm{k}}$, Michael Bauer ${ }^{\mathrm{k}}$, Pascal Sienaert ${ }^{c}$, Sónia Quintão ${ }^{\mathrm{d}, \mathrm{e}}$, Marc-Andreas Edel ${ }^{\mathrm{j}}$, Csilla Bolyos ${ }^{\mathrm{b}}$, Jose Luis Ayuso-Mateos, ${ }^{\mathrm{g}, \mathrm{l}, \mathrm{m}}$, Pilar López-García ${ }^{\mathrm{g}, \mathrm{l}, \mathrm{m}}$, Michael Kluge ${ }^{a}$

\footnotetext{
a Department of Psychiatry and Psychotherapy, University Hospital Leipzig, Leipzig, Germany ${ }^{\mathrm{b}}$ Department of Psychiatry and Psychotherapy, Semmelweis University, Budapest, Hungary 'University Psychiatric Center KU Leuven, Leuven, Belgium

${ }^{\mathrm{d} D e p a r t a m e n t o ~ d e ~ P s i q u i a t r i a, ~ H o s p i t a l ~ d e ~ E g a s ~ M o n i z, ~ C e n t r o ~ H o s p i t a l a r ~ d e ~ L i s b o a ~ O c i d e n t a l, ~ L i s b o n, ~ P o r t u g a l ~}$ 'ISPUP, Instituto de Saúde Pública, Universidade do Porto, Porto, Portugal

${ }^{f}$ Department of Psychiatry, Hospital Santiago Apostol, Vitoria, Spain

${ }^{9}$ Centro de Investigación Biomédica en Red de Salud Mental. CIBERSAM, Madrid, Spain

hepartment of Psychiatry, Hospital del Mar, Barcelona, Spain

'Department of Psychiatry, Hospital Clinic, University of Barcelona, IDIBAPS, Barcelona, Spain

${ }^{j}$ Department of Psychiatry and Psychotherapy, University of Bochum, Bochum, Germany

${ }^{\mathrm{k}}$ Department of Psychiatry and Psychotherapy, Technische Universität Dresden, Dresden, Germany

'Department of Psychiatry, Universidad Autonoma de Madrid, Madrid, Spain

$\mathrm{m}$ Instituto de Investigación Sanitaria Princesa (IP), Madrid, Spain
}

Received 18 January 2017; received in revised form 19 September 2017; accepted 3 November 2017

\footnotetext{
*Correspondence to: Department of Psychiatry and Psychotherapy, University of Leipzig, Semmelweisstr. 10, D-04103 Leipzig, Germany. Fax: +0049 (0)341/97 24539.

E-mail address:
}

Ulrich.Hegerl@medizin.uni-leipzig.de (U. Hegerl). 


\section{KEYWORDS}

Clinical trial; Methylphenidate;

Placebo;

Mania;

Short-term effects

\begin{abstract}
Based on many clinical and preclinical findings the 'vigilance regulation model of mania' postulates that an unstable regulation of wakefulness is a pathogenetic factor in both mania and Attention Deficit Hyperactivity Disorder (ADHD) and induces hyperactivity and sensation seeking as an autoregulatory attempt to stabilize wakefulness. Accordingly, stimulant medications with their vigilance stabilizing properties could have rapid antimanic effects similar to their beneficial effects in ADHD. The MEMAP study - a multi-center, double-blind, placebo-controlled and randomized clinical trial (RCT) - assessed the antimanic efficacy and safety of a 2.5-day treatment with methylphenidate (20-40 mg/day). Of 157 screened patients with acute mania, 42 were randomly assigned to receive $20-40 \mathrm{mg}$ per day of methylphenidate in one or two applications, or placebo. The primary outcome was the change in Young Mania Rating Scale (YMRS) sum scores from baseline to day 2.5 in the methylphenidate group compared to the placebo group. A group sequential design was chosen to justify early RCT termination based on efficacy or futility at an interim analysis after inclusion of 40 patients. In the interim analysis, the change from baseline in the YMRS total score at day 2.5 was not significantly different between both groups $(F(1,37)=0.23 ; p=0.64)$. Thus, futility was declared for methylphenidate and the RCT was stopped. In summary, although methylphenidate was well tolerated and safe in the full analysis set, it failed to show efficacy in the treatment of acute mania. Trial registration: clinicaltrials.gov (URL: http://www.clinicaltrials.gov; registration number: NCT01541605).
\end{abstract} (c) 2017 Elsevier B.V. and ECNP. All rights reserved.

\section{Introduction}

Treatment response of patients with acute mania with antipsychotic agents, benzodiazepines or lithium often requires high dosages and occurs with a delay of several days (Goikolea et al., 2013; Grande et al., 2016). Recently the 'vigilance regulation model of mania' has been proposed which suggests that stimulant medications could be a treatment option similar to their beneficial effects in ADHD (Hegerl et al., 2009; Hegerl and Hensch, 2014). This model is based on a variety of clinical as well as preclinical findings which suggest that an unstable regulation of vigilance (vigilance="brain arousal") is an important pathogenetic factor not only in ADHD but also in mania. Manic symptoms are interpreted as an autoregulatory attempt of the organism to stabilize vigilance by creating a stimulating environment (Hegerl and Hensch, 2014). Indeed, our research group and others have found unstable vigilance regulation with rapid transitions to EEG drowsiness patterns and sleep stages in mania (Van Sweden, 1986; Ulrich, 1994; Small et al., 1999; Schönknecht et al., 2010). Furthermore, destabilizing vigilance (e.g. by sleep deficits or therapeutic sleep deprivation) can trigger mania in vulnerable subjects (Wu and Bunney, 1990; Plante and Winkelman, 2008; for further references see Hegerl and Hensch, 2014); in contrast, stabilizing vigilance (e.g. by prolonged sleep) could be shown to have antimanic effects (e.g., Frank et al., 2005). In line with this concept antimanic effects of stimulant medications have been reported in several case reports and case series (e.g., Beckmann and Heinemann, 1976; Garvey et al., 1987; Schönknecht et al., 2010; for review see Hegerl et al., 2009 as well as Hegerl and Hensch, 2014). A pilot study (Bschor et al., 2001) even demonstrated reduction of manic symptoms already two hours after onset of treatment with methylphenidate in a patient with acute mania and unstable vigilance regulation (for further arguments for antimanic effects of stimulant medications see Hegerl and Hensch, 2014).

In view of this background, the MEMAP study (Kluge et al., 2013), a RCT was designed to assess the efficacy and safety of short-term treatment with methylphenidate in patients with acute mania.

The primary aim of the RCT was to test the hypothesis that a 2.5 day treatment with methylphenidate immediate release given twice daily has better antimanic effects measured with the Young Mania Rating Scale (YMRS, primary outcome) (Young et al., 1978) than placebo. It was further analysed whether instability of vigilance at baseline predicts response to methylphenidate.

\section{Experimental procedures}

\subsection{Study design overview}

Details of the study design have been published elsewhere (Kluge et al., 2013). In short, the MEMAP study is an exploratory, randomized, double-blind, placebo-controlled, international multi-center phase IIIb RCT. It has been designed to assess the efficacy and safety of the stimulant medication drug methylphenidate (Medikinet $\AA$ ) in the initial 2.5 day treatment of acute mania in patients suffering from bipolar affective disorders. The primary comparison in this RCT was between methylphenidate immediate release given twice daily per os $(15 \mathrm{mg}$ at $10 \mathrm{a} . \mathrm{m}$. and 3 p.m. on the first day of treatment (day 1), $20 \mathrm{mg}$ at 9 a.m. and 3 p.m. on day 2 and $20 \mathrm{mg}$ at 9 a.m. on day 3 ) and placebo (also given twice daily). The lower dose of methylphenidate on day 3 was due to the restriction of treatment duration to 2.5 days and thus a single application at 9 a.m. The patients got the drug only 
in the morning at this day because the examinations had been terminated about noon. All patients in the methylphenidate arm received the same dosage at the same time points. Medications could not be added or adjusted during the RCT. We utilized a group sequential design to be able to stop the study early for clinical utility or futility based on a pre-specified interim analysis. The participants of the study were recruited at centers in Belgium, Germany, Hungary and Spain (for further details see Kluge et al., 2013).

\subsection{Setting and participants}

Patients included were adult inpatients, competent to agree to study participation and able to give written informed consent. They had to present with a manic episode according to ICD-10 (Dilling et al., 2006) criteria (F30.0, F30.1, F31.0 or F31.1), a YMRS sum score of at least 20 and maximally 45 points at screening, a body mass index $(\mathrm{BMI})>17$ and able to swallow tablets thus being able to take the study drugs (see Kluge et al., 2013).

Exclusion criteria were: any other current major psychiatric disorder classified using ICD-10 criteria with the exception of attention deficit hyperactivity disorder (ADHD) or other hyperkinetic disorders, harmful tobacco use, neurotic, stress-related and somatoform disorders, behavioral syndromes related to physiological disturbances and physical factors and personality disorders; psychotic features; contraindications for taking methylphenidate (like hypersensitivity to components of this drug, very pronounced anxiety and agitation, ineffective contraception during the conduct of the RCT); severe non-psychiatric diseases (e.g., metabolic disorders) interfering with the aims of the RCT or with individual safety or compliance, as evaluated by the study investigator; oral intake of MAO-inhibitors within 14 days, fluoxetine within six weeks and of any other antidepressant drugs or primarily psychotropic substances within one week before study entry; stable treatment with mood stabilizers like lithium, anticonvulsants (e.g. valproate, carbamazepine) or neuroleptics (e.g. risperidone) or benzodiazepines was not an exclusion criterion and was continued; however, patients taking more than two of these substances could not be selected for inclusion; medical history of cardiovascular diseases, serious hypertension, glaucoma and hyperfunction of the thyroid; long QT syndrome or a family history of QT prolongation, sudden cardiac death or other significant inherited cardiovascular disorders; a history of electroconvulsive therapy within the last three months; known alcohol and drug dependency or abuse (except for patients with an abstinence exceeding three months) with sporadic abuse of cannabis not being an exclusion criterion; pregnancy or nursing; concomitant participation in another clinical trial or participation during the 30 days before screening; prior participation in this RCT or suicidality (for a more detailed summary see Kluge et al., 2013).

Physical health was judged by the investigator after a physical examination. A drug screening was performed by using urine analysis.

Patients were randomly assigned in a 1:1 ratio to receive either 20-40 mg per day of methylphenidate immediate release in one or two applications or placebo administered twice daily. The study protocol had been approved by the leading Ethics Committees and Health Authorities in Germany, Hungary and Spain and the responsible Health Authority in Belgium (Kluge et al., 2013). The RCT was conducted according to the guidelines laid down in the Helsinki Declaration of 1975 and in accordance with the International Conference on Harmonization (ICH) - Good Clinical Practice (GCP) guidelines for clinical trials (see also Kluge et al., 2013). All patients gave written informed consent prior to inclusion in the study.

\subsection{Evaluations of efficacy}

The primary efficacy measure in this study was the severity of manic symptoms as measured by a clinician-administered mania rating scale, the Young Mania Rating Scale (YMRS) (Young et al., 1978). A YMRS sum score of at least 12 points reflects mania (for further details see Kluge et al., 2013). The primary endpoint was determined after 2.5 days of treatment with methylphenidate or placebo.

The secondary efficacy measures were as follows (see Kluge et al., 2013):

- Severity of agitation as measured by the Positive and Negative Syndrome Scale - excited component (PANSS-EC) (Montoya et al., 2011) (administered at baseline, second day of treatment (day 2) and last day of treatment (day 3));

- Clinical impressions as measured by using the Clinical Global Impressions Scale Bipolar version (CGI-BP) (Spearing et al., 1997) rated twice (before the first and after the last treatment with methylphenidate or placebo);

- Cognitive performance as measured by the "Screen for Cognitive Impairment in Psychiatry" (SCIP) (Purdon, 2005) (administered at baseline, day 2 and day 3 (see above));

- EEG-vigilance during a 15-min resting EEG recorded twice, before the first and after the last treatment with methylphenidate or placebo (see appendix 1 for details concerning the EEG analysis);

- Total amount of movements over the study period as measured by actigraphy.

\subsection{Evaluations of safety}

Evaluation of safety comprised the measurement of vital signs (systolic and diastolic blood pressure, heart rate), monitoring of the cardiac function by a 12-lead electrocardiogram (ECG), clinical laboratory tests (for details see Kluge et al., 2013) as well as monitoring of adverse events.

Adverse events were registered in the afternoon of each visit by asking the patient to answer non-leading questions like: "Have you had any health problems today/since you were last asked?" (Kluge et al., 2013).

\subsection{Statistical analysis}

A sample size calculation (for details see Kluge et al., 2013) revealed that 44 patients per treatment group would be required to enable us to find a statistically significant result. Thus, 88 patients should be recruited for the study. However, since the empirical basis for the power analysis was small, it was decided to perform an adaptive interim analysis after inclusion of about 20 patients per treatment arm following some rules specified by Bauer and Köhne (1994):

- Early stopping because of futility, meaning that it is unlikely that the null hypothesis can be rejected by recruiting more patients $\left(p_{1} \geq 0.30\left(\alpha_{0}\right)\right)$;

- Early stopping due to rejection of the null hypothesis $\left(p_{1} \leq 0.03\right.$ $\left.\left(\alpha_{1}\right)\right)$;

- Re-estimation of the sample size in case of study continuation (if $\mathrm{p}_{1}$ was within the interval $\left.\left(\alpha_{1}, \alpha_{0}\right)\right)$.

Prior to statistical analyses, two populations had been defined (see Kluge et al., 2013):

- Full analysis set: This population consists of all randomized patients, who receive at least one dose of treatment regardless of violations of the study protocol. Unless otherwise noted, all analyses were conducted on this population corresponding to an Intent-to-Treat (ITT) population, with the last observations carried forward (LOCF).

- Per protocol (PP) population: This population consists of all patients from the Full analysis set who completed the RCT and did 
not show any major protocol violations. Secondary analyses on the PP analysis set were restricted to the primary outcome.

Analysis of covariance (ANCOVA) was computed in order to analyze differences between methylphenidate and placebo regarding the primary outcome (the mean change from baseline in the sum score of the YMRS at day 2.5), with gender, age and the severity of mania (as reflected by the YMRS total score at baseline) being the covariates. Regarding secondary efficacy outcomes, analogous ANCOVA models were applied. Regarding actigraphic parameters, Mann-Whitney $U$ tests were chosen in view of the rather low sample sizes and/or nonnormal distribution of the dependent variables as assessed by the Kolmogorov-Smirnov test $(p<0.05)$.

Using Spearman-Brown correlation coefficients, the association of the lability index (for details see Appendix 1) at baseline and changes of the severity of mania (as reflected by differences between YMRS sum scores (baseline - day 3)) was analyzed for patients in the methylphenidate group and patients in the placebo group separately. For effect size calculations, Cohen's $d$ and the corresponding $95 \%$ confidence intervals $(\mathrm{Cl})$ were computed.

For safety analyses, descriptive statistics were applied.

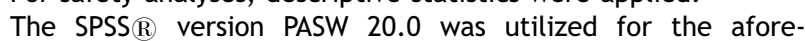
mentioned statistical tests and the significance level was set at $\alpha=0.05$. All statistical tests were two-sided.

\section{Results}

\subsection{Participant disposition and patient characteristics}

Participant disposition is illustrated in Fig. 1.

The interim analysis was performed after 42 patients had been accrued. Of these patients, all were analyzable for efficacy. Overall, there were no significant between-group differences in demographic and clinical characteristics of patients at baseline (see Table 1). Group differences regarding comedication were more pronounced (see Table S1). However, patients from the methylphenidate group and patients in the group getting a placebo did not significantly differ in the proportion of patients with concomitant medication $\left(40.9 \%(9 / 22)\right.$ versus $45.0 \%(9 / 20) ; \chi^{2}=0.67$; $\mathrm{df}=1 ; p=0.41)$. Moreover, there were no significant group differences in the number of individuals taking drugs from a certain class and in the mean number of drugs from this class per patient (see Table S1

\subsection{Results of the interim analysis}

The results for the primary efficacy outcome (YMRS, analysis of covariance (ANCOVA) with YMRS baseline sum scores, age and gender as covariates are as follows (see Fig. 2).

The change from baseline in the YMRS total score at day 2.5 after baseline was not significantly different between both groups $(F(1,37)=0.23 ; p=0.64$; difference from placebo: -4.50 points; effect size (Cohen's d): $-0.48 ; 95 \%$ confidence interval $(\mathrm{Cl}):-1.08$ to 0.14$)$. Given this result and the fact that the two-sided $p$ value $(0.64)$ clearly surpassed the $p$ value boundary $\left(p_{1}=0.30\right)$ for rejection of the alternative hypothesis (methylphenidate is significantly superior to placebo), futility was declared for methylphenidate and the RCT was stopped.

Analysis on the Per Protocol analysis set which consisted of all patients who completed the study and did not have any major violations of the study protocol, was conducted for the primary efficacy outcome, too. It supported the finding that the methylphenidate group and the placebo group did not significantly differ regarding the change from baseline in the YMRS total score at day 2.5 after baseline (mean change in YMRS total score from baseline (8 a.m.) (S.D.): 5.07 (11.44) in the methylphenidate group $(n=15)$ versus $7.82(6.42)$ in the placebo group $(n=17)$; difference from placebo: -2.75 points; effect size (Cohen's d): $-0.30 ; 95 \% \mathrm{Cl}$ : -0.99 to 0.40 ;



Fig. 1 CONSORT diagram of subject progress through the phases of the MEMAP trial. 
Table 1 Characteristics of the sample $(N=42)$.

\begin{tabular}{|c|c|c|c|}
\hline Variables & Methylphenidate $(N=22)$ & Placebo $(N=20)$ & $\mathrm{p}$ value (2-sided) \\
\hline Age (mean (S.D.)) & $43.32(12.16)$ & $49.10(15.72)$ & $0.19^{a}$ \\
\hline Gender (Males (\%)) & $13 / 22(59.1 \%)$ & $9 / 20(45 \%)$ & $0.36^{\mathrm{b}}$ \\
\hline Ethnicity (Caucasian) (\%) & $21 / 22(95.5 \%)$ & $20 / 20(100 \%)$ & $1.00^{c}$ \\
\hline Duration of illness (years) (mean (S.D.)) & $17.41(11.92)$ & $21.74(15.00)[N=19]$ & $0.31^{\mathrm{a}}$ \\
\hline $\begin{array}{l}\text { Age at the first depressive episode (years) } \\
\text { (mean (S.D.)) }\end{array}$ & $21.26(14.54)[N=19]$ & $23.29(17.16)[N=17]$ & $0.70^{\mathrm{a}}$ \\
\hline Age at the first manic episode (years) (mean (S.D.)) & $28.27(13.40)$ & $30.95(15.36)[N=19]$ & $0.56^{\mathrm{a}}$ \\
\hline Age at first treatment (years) (mean (S.D.)) & $28.41(13.20)$ & $26.16(14.82)[N=19]$ & $0.61^{a}$ \\
\hline $\begin{array}{l}\text { Duration of the current manic episode (days) } \\
\text { (mean (S.D.)) }\end{array}$ & $11.14(15.96)[N=21]$ & $11.57(10.75)[N=14]$ & $0.93^{\mathrm{a}}$ \\
\hline Number of depressive episodes (mean (S.D.)) & $3.00(4.61)$ & $2.53(3.12)[N=19]$ & $0.96^{d}$ \\
\hline Number of manic episodes (mean (S.D.)) & $5.50(6.15)$ & $4.89(3.82)[N=18]$ & $0.89^{d}$ \\
\hline Number of mixed episodes (mean (S.D.)) & $0.55(2.13)$ & $0.68(2.11)[N=19]$ & $0.80^{d}$ \\
\hline Current psychotropic medication (\%) & $20 / 22(90.9 \%)$ & $14 / 20(70.0 \%)$ & $0.12^{c}$ \\
\hline Baseline YMRS total score (8 a.m.) (mean (S.D.)) & $24.95(6.51)$ & $27.55(6.34)$ & $0.20^{\mathrm{a}}$ \\
\hline Total CGI-BP score at baseline & $4.77(0.87)$ & $4.95(1.05)$ & $0.34^{\mathrm{d}}$ \\
\hline
\end{tabular}

CGI-BP=Clinical Global Impression-Bipolar Scale (Spearing et al., 1997); S.D.=standard deviation; YMRS=Young Mania Rating Scale (Young et al., 1978).

${ }^{a}$ According to the results of a t test for independent sample comparisons.

${ }^{b}$ According to the results of a chi-square test for two-by.-two cross tables.

${ }^{c}$ According to the results of Fisher's exact test for two-by-two cross tables.

${ }^{d}$ According to the results of a Mann-Whitney $U$ test (in cases of non-normal distribution of the dependent variable as assessed by the Kolmogorov-Smirnov test $(p<0.05))$.

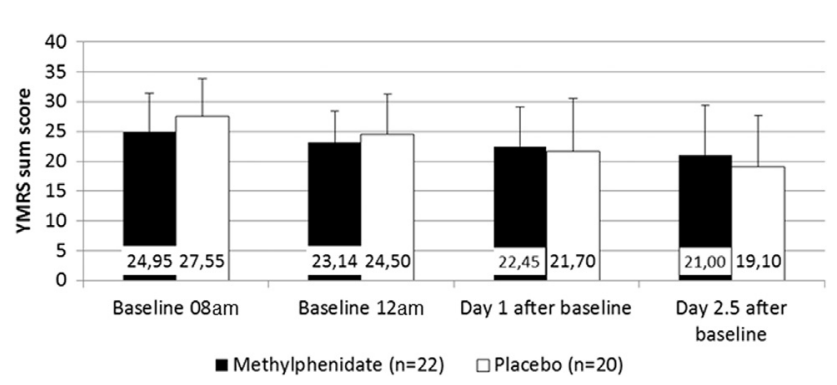

Fig. 2 Results of the primary statistical analysis based on the interim analysis database for a randomized clinical trial comparing acute anti-manic effects of methylphenidate with placebo $(N=42)$. The mean change in the YMRS total score from baseline (baseline 8 a.m. - day 2.5 after baseline) was 3.95 points (standard deviation (S.D.) $=10.79$ points) in the methylphenidate group and 8.45 points (S.D. $=7.59$ points) in the placebo group. YMRS = Young Mania Rating Scale (Young et al., 1978). Error bars represent standard deviations.

$F(1,27)=0.01 ; p=0.92)$. The same was true for short-term changes of manic symptoms within four hours (measured by the YMRS) (mean baseline score at 8 a.m. (S.D.): 24.95 (6.51) in the methylphenidate group versus 27.55 (6.34) in the placebo group; mean change in YMRS total score from baseline (8 a.m.) (S.D.): 1.82 (4.94) in the methylphenidate group versus 3.05 (4.68) in the placebo group; difference from placebo: -1.23 points; effect size (Cohen's $d)$ : -0.26 ; $95 \% \mathrm{Cl}:-0.86$ to $0.36 ; \mathrm{F}(1,39)=0.07 ; p=0.80)$.
Secondary analyses of scores on the PANSS-EC, CGI-BP and SCIP demonstrated lack of efficacy for methylphenidate on these endpoints (see Table 2), too.

The association between the vigilance lability index at baseline and improvement of the YMRS total scores at day 2.5 was positive and moderately high in the methylphenidate group ( $r h o=0.47 ; p=0.15 ; n=11)$; however, a positive association between these variables was also found in the placebo group (rho=0.60; $p=0.21 ; n=6$ ) (see Fig. 3 ).

Overall, there were no significant differences between methylphenidate and placebo regarding EEG vigilance parameters $(p \geq 0.27$; see Table S2).

Changes in motor activity over the study period did not differ between methylphenidate and placebo (see Table 2).

\subsection{Safety results}

Vital parameters (blood pressure and heart rate) did not differ significantly between the methylphenidate group and the placebo group regarding changes from baseline (see Table 2).

Adverse events reported in more than one patient in the methylphenidate group included worsening of mania $(n=2)$ and headache $(n=2)$. Further adverse events in the methylphenidate group were: worsening of arterial hypertension $(n=1)$, discrete paraesthesia on the scalp $(n=1)$, increase of an already existing tardive dyskinesia $(n=1)$, facial edema and stiffness $(n=1)$, rough skin of both hands $(n=1)$ and general pain $(n=1)$. 
Table 2 Secondary outcomes.

\begin{tabular}{|c|c|c|c|c|}
\hline Variables (mean (S.D.)) & Methylphenidate $(N=22)$ & Placebo $(N=20)$ & Effect size (Cohen's d) $(95 \% \mathrm{Cl})$ & $p$ Value (two-sided) \\
\hline \multicolumn{5}{|l|}{ PANSS-EC } \\
\hline Baseline PANSS-EC total score ( 8 a.m.) & $14.27(4.04)$ & $16.40(4.98)$ & - & - \\
\hline Total PANSS-EC score at baseline (12 a.m.) & $12.41(3.80)$ & $13.20(5.45)$ & - & - \\
\hline Change in PANSS-EC total score from 8 a.m. to 12 a.m. & $1.86(3.85)$ & $3.20(5.05)$ & $-0.30(-0.90$ to 0.31$)$ & $0.77^{\mathrm{a}}$ \\
\hline Total PANSS-EC score at baseline & $14.27(4.04)$ & $16.40(4.98)$ & - & - \\
\hline Total PANSS-EC score at day 2.5 after baseline & $11.81(4.88)[n=21]$ & $10.85(4.75)$ & - & - \\
\hline Change in PANSS-EC total score from baseline & $2.33(5.88)[n=21]$ & $5.55(5.31)$ & $-0.57(-1.19$ to 0.06$)$ & $0.28^{b}$ \\
\hline \multicolumn{5}{|l|}{ CGI-BP } \\
\hline Total CGI-BP score at baseline & $4.77(0.87)$ & $4.95(1.05)$ & - & - \\
\hline Total CGI-BP score at day 2.5 after baseline & $4.43(0.75)[n=21]$ & $3.95(1.43)$ & - & - \\
\hline Change in CGI-BP total score from baseline & $0.33(1.02)[n=21]$ & $1.00(1.34)$ & $-0.56(-1.18$ to 0.07$)$ & $0.10^{c}$ \\
\hline \multicolumn{5}{|l|}{ SCIP } \\
\hline \multicolumn{5}{|l|}{ List learning test } \\
\hline Total score at baseline & $19.91(5.77)$ & $18.05(5.17)$ & - & - \\
\hline Total score at day 2.5 after baseline & $18.67(4.41)[n=21]$ & $17.95(4.66)$ & - & - \\
\hline Change in score from baseline & $1.71(3.69)[n=21]$ & $0.10(3.49)$ & $0.45(-0.18$ to 1.06$)$ & $0.44^{d}$ \\
\hline \multicolumn{5}{|l|}{ Consonant repetition test } \\
\hline Total score at baseline & $17.91(4.62)$ & $16.00(4.01)$ & - & - \\
\hline Total score at day 2.5 after baseline & $19.52(4.25)[n=21]$ & $17.55(4.61)$ & - & - \\
\hline Change in score from baseline & $-1.57(2.42)[n=21]$ & $-1.55(3.07)$ & $-0.01(-0.62$ to 0.61$)$ & $0.65^{d}$ \\
\hline \multicolumn{5}{|l|}{ Verbal fluency test } \\
\hline Total score at baseline & $15.68(7.69)$ & $17.35(8.07)$ & - & - \\
\hline Total score at day 2.5 after baseline & $18.62(8.10)[n=21]$ & $18.45(7.88)$ & - & - \\
\hline Change in score from baseline & $-2.48(5.48)[n=21]$ & $-1.10(5.12)$ & $-0.26(-0.87$ to 0.36$)$ & $0.49^{d}$ \\
\hline \multicolumn{5}{|l|}{ Delayed list learning test } \\
\hline Total score at baseline & $5.36(2.82)$ & $4.65(3.27)$ & - & - \\
\hline Total score at day 2.5 after baseline & $4.14(2.24)[n=21]$ & $3.40(2.66)$ & - & - \\
\hline Change in score from baseline & $1.33(1.96)[n=21]$ & $1.25(2.10)$ & $0.04(-0.57$ to 0.65$)$ & $0.64^{d}$ \\
\hline \multicolumn{5}{|l|}{ Visuomotor tracking test } \\
\hline Total score at baseline & $8.95(3.84)$ & $8.05(4.22)$ & - & - \\
\hline Total score at day 2.5 after baseline & $10.14(4.21)[n=21]$ & $8.85(4.39)$ & - & - \\
\hline Change in score from baseline & $-1.00(1.82)[n=21]$ & $-0.80(2.88)$ & $-0.08(-0.69$ to 0.53$)$ & $0.68^{d}$ \\
\hline \multicolumn{5}{|l|}{ Actigraphic parameters } \\
\hline Mean activity score at baseline & $56.46(12.50)[n=11]$ & $56.44(27.11)[n=11]$ & - & $0.31^{\mathrm{e}}$ \\
\hline Changes in the mean activity score (baseline - day 2 ) & $0.75(7.83)[n=11]$ & $2.58(10.44)[n=11]$ & $-0.20(-1.03$ to 0.65$)$ & $0.58^{\mathrm{e}}$ \\
\hline
\end{tabular}


Diastolic blood pressure

Score at baseline $(\mathrm{mm} \mathrm{Hg})$

Change from baseline $(\mathrm{mm} \mathrm{Hg})$

$81.18(14.46)$

$0.86(18.67)[n=21]$

$83.80(10.15)$

Systolic blood pressure

Score at baseline $(\mathrm{mm} \mathrm{Hg})$

Change from baseline $(\mathrm{mm} \mathrm{Hg})$

$128.50(17.03)$

$4.62(12.70)[n=21]$

$131.70(24.03)$

$11.42(15.87)[n=19] \quad-0.48(-1.10$ to 0.16$)$

\section{Heart rate}

Score at baseline (/minute)

$81.36(15.07)$

$86.35(12.32)$

$-6.24(14.66)[n=21] \quad-0.26(9.64)[n=19] \quad-0.48(-1.10$ to 0.16$)$

CGI-BP=Clinical Global Impression-Bipolar Scale (Spearing et al., 1997); PANSS-EC=Excited Component of the Positive and Negative Syndrome Scale (Montoya et al., 2011); SCIP=Screen for Cognitive Impairment in Psychosis (Purdon, 2005); S.D.=standard deviation.

aBased on an analysis of covariance (ANCOVA) with the PANSS-EC total score at the first day few hours after baseline as dependent variable, "group" $(1=$ Methylphenidate; $0=$ Placebo) as independent variable as well as the PANSS-EC total score at baseline $(8 \mathrm{a} . \mathrm{m}$.) as covariate.

bBased on an analysis of covariance (ANCOVA) with the PANSS-EC total score at day 2.5 after baseline as dependent variable, "group" (1=Methylphenidate; $0=$ Placebo) as independent variable as well as the PANSS-EC total score at baseline $(8$ a.m.) as covariate.

"Based on an analysis of covariance (ANCOVA) with the CGI-BP total score at day 2.5 after baseline as dependent variable, "group" ( $1=$ Methylphenidate; $0=$ Placebo) as independent variable as well as the CGI-BP total score at baseline as covariate.

'Based on an analysis of covariance (ANCOVA) with the score in one of the five SCIP subtests at day 2.5 after baseline as dependent variable, "group" (1=Methylphenidate; $0=$ Placebo) as independent variable as well as the score in the same SCIP subtest at baseline as covariate.

eaccording to the results of a Mann-Whitney $\mathrm{U}$ test (in cases of low sample size/non-normal distribution of the dependent variable as assessed by the Kolmogorov-Smirnov test $(p<0.05))$.

fBased on an analysis of covariance (ANCOVA) with the vital parameter (e.g., heart rate) at day 2 after baseline as dependent variable, "group" ( $1=$ Methylphenidate; $0=$ Placebo) as independent variable as well as the score in the same vital parameter at baseline as covariate. 

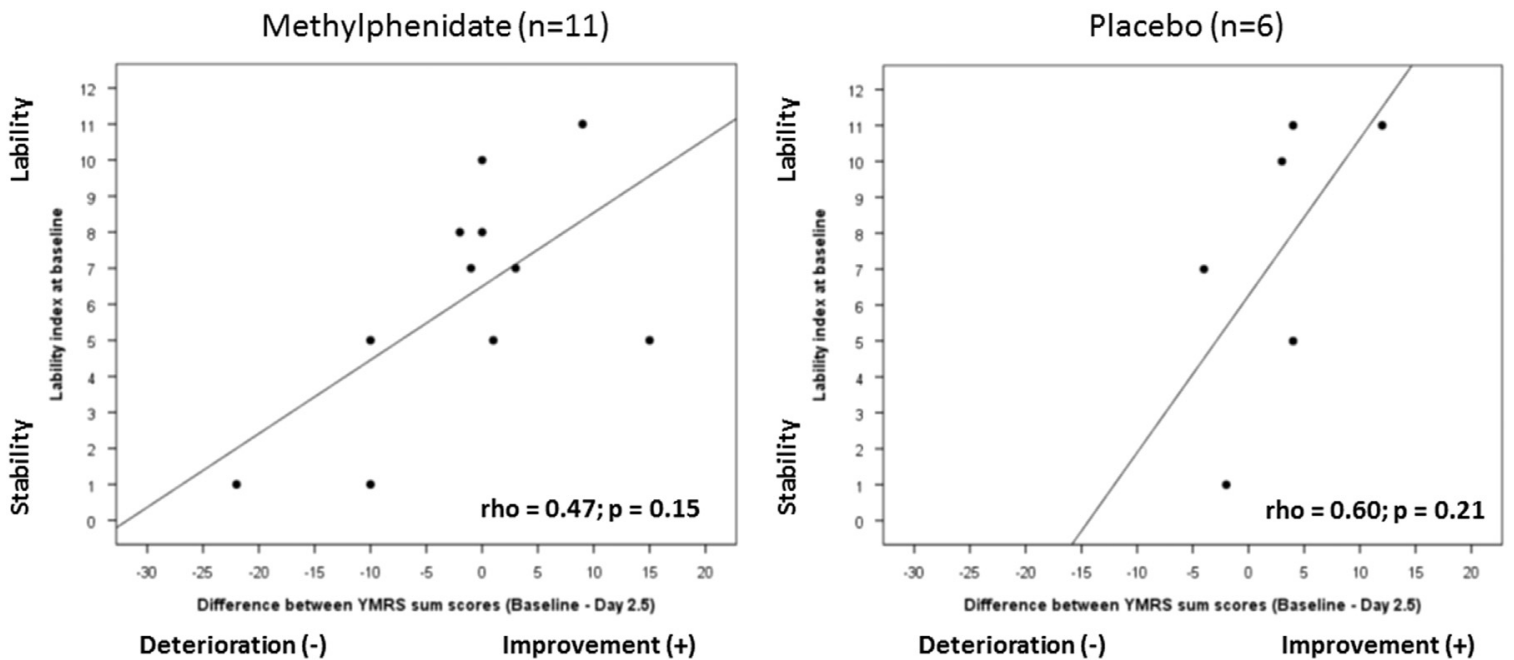

Fig. 3 Association of the lability score at baseline and changes of the severity of manic symptoms in patients treated with methylphenidate $(n=11)$ and patients from the placebo group $(n=6)$. rho=Spearman-Brown correlation coefficient; YMRS=Young Mania Rating Scale (Young et al., 1978). The stages of the lability index at baseline are as follows: $1=$ unique appearance of the vigilance stages 0 and $A 1$ throughout the recording; 2 =unique appearance of the vigilance stage $A$ throughout the recording; $3=$ vigilance stage $B$ in minutes $11-15 ; 4=$ vigilance stage $B$ in minutes $6-10 ; 5=$ vigilance stage $B$ in minutes $1-5 ; 6=$ vigilance stage $B 23$ in minutes $11-15 ; 7=$ vigilance stage $B 23$ in minutes $6-10 ; 8=$ vigilance stage B23 in minutes $1-5 ; 9=$ sleep stage $C$ in minutes 11 $15 ; 10=$ sleep stage $C$ in minutes $6-10 ; 11=$ sleep stage $C$ in minutes $1-5$.

In the placebo group, the following adverse events were present: worsening of instable mood and lability of the affect $(n=1)$, diarrhea and vomiting $(n=1)$ and worsening of mania $(n=1)$.

There were no serious adverse events during the trial.

One participant in the methylphenidate group retracted his consent because of a potentially treatment-related adverse event (worsening of mania). Three other patients were excluded from the study ex post because of loss to follow-up (one patient from the methylphenidate group), or because of wished discharge of the hospital (one patient from the methylphenidate group, one patient from the placebo group).

\section{Discussion}

The hypothesis that acute short-term treatment with methylphenidate has antimanic effects in patients with bipolar affective disorder was not confirmed. Also concerning secondary outcomes such as cognitive performance (SCIP (Purdon, 2005)), global functioning (CGI-BP (Spearing et al., 1997)) and severity of agitation (PANSS-EC (Montoya et al., 2011)) no group differences were found.

It was hypothesized that there might be a causal relationship between the instability of vigilance regulation and response to methylphenidate. However, although it seemed as if there was a trend toward this relationship in the methylphenidate group, this could not be confirmed since a similar treatment response was observed in the placebo group.

Methylphenidate treatment was not associated with a reduction of motor activity as assessed by actigraphy; however, this finding is based only on a small subsample of patients.

Overall, methylphenidate was a safe drug and well tolerated in patients with acute mania.
Some methodological limitations should be discussed: first, the inclusion and exclusion criteria of the MEMAP trial were rather strict; therefore, the generalizability of the study findings is clearly limited. Second, the selected dosages of methylphenidate (day 1: $30 \mathrm{mg} / \mathrm{day}$, day 2: $40 \mathrm{mg} /$ day; day 3: $20 \mathrm{mg} /$ day) might have been too low, the group differences regarding comedication too pronounced and treatment duration too short in order to make clinically relevant anti-manic effects possible. A dosage of $40 \mathrm{mg} /$ day was reached only at the penultimate day. In adult patients with ADHD, which shows several similarities to mania at the symptomatic level (see Hegerl and Hensch, 2014), often higher dosages are needed in order to see a clear clinical effect (Huss et al., 2014). In adult patients with $A D H D$ a dose range of $40-80 \mathrm{mg}$ methylphenidate per day is recommended (Huss et al., 2014). According to this study marked clinical effects of methylphenidate in this dose range exceeding those of a placebo were only present at treatment week 5 . Thus, although many manic patients report rapid-onset antimanic effects of methylphenidate there are study findings showing strongest clinical effects of methylphenidate only after five weeks. This raises the question whether or not the treatment duration of $2 \frac{1}{2}$ days was too short to see relevant effects.

Thus, it would be worthwhile to design a RCT with higher doses of methylphenidate for the initial treatment of acute mania. The reasons for choosing a relatively low dose of methylphenidate and the short duration of the trial were basically ethical, given the theoretical risk of worsening mania by giving a dopamine agonist to manic patients. In view of the results of this proof-of-concept trial, the safety of methylphenidate would now justify a trial with higher doses and longer duration. However, our study does not support a rapid-onset effect of any anti-manic action of methylphenidate as postulated previously (Bschor et al., 2001; Hegerl and Hensch, 2014). Moreover, our study had a 
rather small sample size. In addition, it cannot be excluded that different concomitant drugs had a certain influence on differences between methylphenidate and placebo regarding anti-manic efficacy.

In summary, the MEMAP trial revealed that methylphenidate (20-40 mg/day per os) despite being well tolerated and safe failed to show efficacy in the initial treatment of acute mania. However, in view of the low dosage and short duration of this RCT it would be premature to exclude anti-manic efficacy of methylphenidate in mania. In an area of psychiatry with huge unmet needs (Fountoulakis et al., 2006), exploring innovative hypotheses and treatments in an ethical framework is of paramount importance.

\section{Role of funding source}

The work was supported by a grant of the Spanish 'Ministerio de Sanidad, Politica Social e Igualdad' (Ministry of Health, Social Politics and Equality), EC10-110, EC10-297, EC10-333 and EC10-064, and by the Instituto de Salud Carlos III of Spain (CIBERSAM), grant 11 INT2 which had been provided after a peer review process. The funding source had no rule in the study design, in the collection, analysis and interpretation of data, in the writing of the manuscript and in the decision to submit the paper for publication.

\section{Conflicts of interest}

Professor Hegerl has served as an advisory board member for Eli Lilly, Lundbeck, Otsuka, Takeda, and Servier, as a consultant for Nycomed, and as a speaker for Bristol-Myers Squibb, Medice Arzneimittel, Novartis, and Roche Pharma. Dr. Dr. Bauer has received grant support from the Stanley Medical Research Institute, NARSAD and the European Commission (FP7). Moreover, he was/is consultant for AstraZeneca, Eli Lilly, Servier, Lundbeck, Bristol-Myers Squibb and Otsuka. He has received Speaker Honoraria from AstraZeneca, Eli Lilly, GlaxoSmithKline, Lundbeck, Bristol-Myers Squibb, Servier and Otsuka. István Bitter reports grants and personal fees from E. Lilly, personal fees from EGIS, Janssen/Janssen-Cilag, Lundbeck, Medavante, Gedeon Richter, Servier, all outside the submitted work. Dr. Edel has received Speaker Honoraria from Medice. Dr. González-Pinto has received grant support, acted as consultant, or given presentations for Almirall, AstraZeneca, Bristol-Myers Squibb, Otsuka, Eli Lilly, Glaxo-SmithKline, Janssen-Cilag, SanofiAventis, Lundbeck, Novartis, Organon, Schering-Plough, the Spanish Ministry of Science and Innovation (CIBERSAM), the Department of Health of the Basque Government, the University of the Basque Country and Pfizer. Dr. Kluge has received honoraria from Roche, Servier and Trommsdorff. Dr. Mergl has served as a consultant for Nycomed, a Takeda company. Dr. Vieta has received grants and has served as a consultant, advisor or CME speaker for Almirall, AstraZeneca, Bristol-Myers Squibb, Cephalon, Eli Lilly, the Forest Research Institute, Gedeon Richter, Glaxo-SmithKline, Janssen-Cilag, Jazz, Johnson \& Johnson, Lundbeck, Merck, Novartis, Organon, Otsuka, Pfizer, Pierre-Fabre, Qualigen, Roche, Sanofi-Aventis, Servier, Shering-Plough, Solvay, Takeda, Teva, the Spanish Ministry of Science and Innovation (CIBERSAM), the Seventh European Framework Programme (ENBREC), the Stanley Medical Research Institute, United Biosource Corporation, and Wyeth. Since 2013, Dr. Ulrich Zimmermann reveived compensation for talks from Lundbeck,
Sächsisches Krankenhaus Rodewisch, Asklepios Klinikum Wiesen, Klinik Hohe Mark, Oberursel, Helios Klinikum Freital, Sächsische Landesärztekammer, Helios Klinikum Plauen, Städtisches Krankenhaus Chemnitz and Klinikum Ingolstadt. He also received conference attendance support from Lundbeck. The present work is unrelated to the above relationships. All other authors declare that they have no conflicts of interest.

\section{Contributors}

$\mathrm{UH}, \mathrm{EV}$ and $\mathrm{MK}$ designed the study and wrote the protocol. UH and RM drafted the manuscript and completed a first version of the article. RM undertook the statistical analyses. Acquisition and analyses of EEG data were performed by CS and JD. In addition, CS acquired and analyzed actigraphic data. AGA, IZ and MK played an important role in the acquisition of clinical data. All authors contributed to interpretations of the results of the MEMAP trial, revised the manuscript and approved the final version of the paper.

\section{Acknowledgements}

The work was supported by a grant of the Spanish "Ministerio de Sanidad, Politica Social e Igualdad' (Ministry of Health, Social Politics and Equality), EC10-110, EC10-297, EC10-333 and EC10-064, and by the Instituto de Salud Carlos III of Spain (CIBERSAM), grant 11INT2 which had been provided after a peer review process. The funding source had no rule in the study design, in the collection, analysis and interpretation of data, in the writing of the manuscript and in the decision to submit the paper for publication.

Study drug was kindly provided by Medice, Iserlohn, Germany. Training videos for training on the YMRS were kindly provided by Bracket, Wayne, USA. We want to thank Jens Dietzel and Ines Thomas for the visual classification of resting EEG vigilance stages in the context of this RCT. Moreover, we want to thank Petra Neuhaus for her contributions to data monitoring and safety analyses and Itziar Leal and Herminio Martinez for their contributions to patients' recruitment. The participating patients are gratefully acknowledged for their willingness to participate in this study.

\section{Appendix A. Supplementary material}

Supplementary data associated with this article can be found in the online version at https://doi.org/10.1016/j. euroneuro.2017.11.003.

\section{References}

Bauer, P., Köhne, K., 1994. Evaluation of experiments with adaptive interim analyses. Biometrics 50, 1029-1041.

Beckmann, H., Heinemann, H., 1976. D-Amphetamine in manic syndrome. Arzneim.-Forsch./Drug Res. 26, 1185-1186.

Bschor, T., Müller-Oerlinghausen, B., Ulrich, G., 2001. Decreased level of EEG-vigilance in acute mania as a possible predictor for a rapid effect of methylphenidate: a case study. Clin. Electroencephalogr. 32, 36-39.

Dilling, H., Mombour, W., Schmidt, M.H., 2006 International Classification of Mental Disorders. ICD-10 Chapter V (F). Diagnostic Criteria for Research and Practice, fourth ed. Huber, Bern.

Fountoulakis, K.N., Vieta, E., Young, A., Yatham, L., Grunze, H., Blier, P., Möller, H.-J., Kasper, S., 2006. The International College of Neuropsychopharmacology (CINP) treatment guidelines for bipolar disorder in adults (CINP-BD-2017), Part 4: unmet needs in the treatment of bipolar disorder and recommendations 
for future research. Int. J. Neuropsychopharmacol. 27. http: //dx.doi.org/10.1093/ijnp/pyw072 (e-pub Print).

Frank, E., Kupfer, D.J., Thase, M.E., Mallinger, A.G., Swartz, H.A., Fagiolini, A.M., Grochocinski, V., Houck, P., Scott, J., Thompson, W., Monk, T., 2005. Two-year outcomes for interpersonal and social rhythm therapy in individuals with bipolar I disorder. Arch. Gen. Psychiatry 62, 996-1004.

Garvey, M.J., Hwang, S., Teubner-Rhodes, D., Zander, J., Rhem, C., 1987. Dextroamphetamine treatment of mania. J. Clin. Psychiatry 48, 412-413.

Goikolea, J.M., Colom, F., Capapey, J., Torres, I., Valenti, M., Grande, I., Undurraga, J., Vieta, E., 2013. Faster onset of antimanic action with haloperidol compared to second-generation antipsychotics: a meta-analysis of randomized clinical trials in acute mania. Eur. Neuropsychopharmacol. 23, 305-316.

Grande, I., Berk, M., Birmaher, B., Vieta, E., 2016. Bipolar disorder. Lancet 387, 1561-1572.

Hegerl, U., Hensch, T., 2014. The vigilance regulation model of affective disorders and ADHD. Neurosci. Biobehav. Rev. 44, 45-57.

Hegerl, U., Sander, C., Olbrich, S., Schönknecht, P., 2009. Are psychostimulants a treatment option in mania? Pharmacopsychiatry 42, 169-174.

Huss, M., Ginsberg, Y., Tvedten, T., Arngrim, T., Philipsen, A., Carter, K., Chen, C.W., Kumar, V., 2014. Methylphenidate hydrochloride modified-release in adults with attention deficit hyperactivity disorder: a randomized double-blind placebo-controlled trial. Adv. Ther. 31, 44-65.

Kluge, M., Hegerl, U., Sander, C., Dietzel, J., Mergl, R., Bitter, I., Demyttenaere, K., Gusmão, R., Gonzalez-Pinto, A., Sola, V.P., Vieta, E., Juckel, G., Zimmermann, U.S., Bauer, M., Sinaert, P., Quintão, S., Edel, M.-A., Bolyos, C., Ayuso-Mateos, J.L., LópezGarcia, P., 2013. Methylphenidate in Mania Project (MEMAP): study protocol of an international randomised double-blind placebo-controlled study on the initial treatment of acute mania with methylphenidate. BMC Psychiatry 13, 71.

Montoya, A., Valladares, A., Lizan, L., San, L., Escobar, R., Paz, S., 2011. Validation of the Excited Component of the Positive and Negative Syndrome Scale (PANSS-EC) in a naturalistic sample of 278 patients with acute psychosis and agitation in a psychiatric emergency room. Health Qual. Life Outcomes 9, 18.

Plante, D.T., Winkelman, J.W., 2008. Sleep disturbance in bipolar disorder: therapeutic implications. Am. J. Psychiatry 165, 830-843.

Purdon, S.E., 2005. The Screen for Cognitive Impairment in Psychiatry (SCIP): Administration Manual and Normative Data. PNL Inc., Edmonton.

Schönknecht, P., Olbrich, S., Sander, C., Spindler, P., Hegerl, U., 2010. Treatment of acute mania with modafinil monotherapy. Biol. Psychiatry 67, e55-e57.

Small, J.G., Milstein, V., Malloy, F.W., Medlock, C.E., Klapper, M.H., 1999. Clinical and quantitative EEG studies of mania. J. Affect. Disord. 53, 217-224.

Spearing, M.K., Post, R.M., Leverich, G.S., Brandt, D., Nolen, W., 1997. Modification of the Clinical Global Impressions (CGI) Scale for use in bipolar illness (BP): the CGI-BP. Psychiatr. Res. 73, 159-171.

Ulrich, G., 1994. Psychiatrische Elektroenzephalographie. Gustav Fischer, Jena.

Van Sweden, B., 1986. Disturbed vigilance in mania. Biol. Psychiatry 21, 311-313.

Wu, J.C., Bunney, W.E., 1990. The biological basis of an antidepressant response to sleep deprivation and relapse: review and hypothesis. Am. J. Psychiatry 147, 14-21.

Young, R.C., Biggs, J.T., Ziegler, V.E., Meyer, D.A., 1978. A rating scale for mania: reliability, validity, and sensitivity. Br. J. Psychiatry 133, 429-435. 